Pacific

Journal of

Mathematics

A FAMILY OF ARITHMETIC SURFACES OF GENUS 3

JORDI GUÀRDIA

Volume $212 \quad$ No. 1

November 2003 


\title{
A FAMILY OF ARITHMETIC SURFACES OF GENUS 3
}

\author{
JORDI GUÀrDIA
}

The aim of this paper is the study of the genus 3 curves

$$
C_{n}: Y^{4}=X^{4}-(4 n-2) X^{2}+1,
$$

from the Arakelov viewpoint. The Jacobian of the curves $C_{n}$ splits as a product of elliptic curves, and this fact gives enough arithmetical datum to determine the stable model and the canonical sheaf of the curves. We use this information to look for explicit expressions of the modular height and the self-intersection of the dualizing sheaf of the curves $C_{n}$.

\section{Introduction.}

The study of a curve from the arithmetical or the Arakelov viewpoints is a hard task, since it involves a very good knowledge of its geometry (differential forms, periods), its arithmetic (locus of bad reduction, stable models) and its analysis (Green function). Classically, two families of curves have been extensively studied: Fermat curves and modular curves. The study of these curves is feasible because they have a large automorphism group. On the other hand, the curves in these families have variable genus. If one wants to study the behaviour of some arithmetical or Arakelov invariants on the moduli space of curves of a given genus, these families are not useful.

The Arakelov invariants of elliptic curves are completely determined $([\mathbf{F a 8 4}])$. Some concrete examples of Arakelov invariants for curves of genus 2 were provided by Bost, Mestre and Moret-Bailly in [Bo-M-M90]. We present here the study of a family of curves of genus 3 .

Let $n \in \mathbb{N}, n \geq 2$ be a natural number such that $n \equiv 2(\bmod 3)$ and $n \not \equiv$ $0,1\left(\bmod 2^{5}\right)$, and consider the projective curve $C_{n}$ given by the equation

$$
Y^{4}=X^{4}-(4 n-2) X^{2} Z^{2}+Z^{4} .
$$

We have studied the geometry of the curves $C_{n}$ in [Gu01]. They are nonsingular curves of genus 3. They have a large group of automorphisms, which gives the chance of performing a great deal of calculations on them. In this article we study the curves $C_{n}$ from the arithmetical and Arakelov viewpoints. We find the stable models of the arithmetic surfaces given by them. Combining both the geometric and the arithmetic information compiled about the curves $C_{n}$, we initiate the study of their Arakelov invariants: Their modular height and the self-intersection of their canonical sheaf. 
The conditions $n \not \equiv 0,1\left(\bmod 2^{5}\right), n \equiv 2(\bmod 3)$ are assumed only for technical reasons, to simplify the exposition. They are necessary essentially for the results about the reduction of the curves $C_{n}$ at primes over 2 , and to avoid some particular cases of supersingularity.

\section{Automorphisms. Splitting of the Jacobian.}

The following results are proved in [Gu01]:

Proposition 2.1. The automorphisms of $C_{n}(n>2)$ are the restrictions of the following projectivities of $\mathbb{P}^{2}(\mathbb{C})$ :

$$
\begin{aligned}
\varphi_{0 k} & =\left(\begin{array}{ccc}
1 & 0 & 0 \\
0 & i^{k} & 0 \\
0 & 0 & 1
\end{array}\right), \varphi_{1 k}=\left(\begin{array}{ccc}
1 & 0 & 0 \\
0 & i^{k} & 0 \\
0 & 0 & -1
\end{array}\right), \\
\varphi_{2 k} & =\left(\begin{array}{ccc}
0 & 0 & 1 \\
0 & i^{k} & 0 \\
1 & 0 & 0
\end{array}\right), \quad \varphi_{3 k}=\left(\begin{array}{ccc}
0 & 0 & 1 \\
0 & i^{k} & 0 \\
-1 & 0 & 0
\end{array}\right),
\end{aligned}
$$

for $k=0,1,2,3$. The automorphisms $\alpha=\varphi_{01}, \beta=\varphi_{12}, \gamma=\varphi_{20}$ form a system of generators for $\operatorname{Aut}\left(C_{n}\right)$, with relations $\alpha^{4}=\beta^{2}=\gamma^{2}=\mathrm{Id}$, $\alpha \beta=\beta \alpha, \alpha \gamma=\gamma \alpha, \beta \gamma=\gamma \beta \alpha^{2}$. The group $\operatorname{Aut}\left(C_{n}\right)$ is isomorphic to a semidirect product $\mathbb{Z} / 4 \mathbb{Z} \ltimes V_{4}$, where $V_{4}$ denotes the Klein group.

Remark. The curve $C_{2}$ has a larger group of automorphisms, because it is isomorphic to the Fermat curve of fourth degree. For our purposes, we only need to know that the above matrices also give automorphisms of $C_{2}$.

The automorphisms $\beta \alpha^{2}, \beta$ and $\alpha^{2}$ provide three elliptic quotients of $C_{n}$. We denote the subgroups that they generate by $G_{1}=\left\langle\beta \alpha^{2}\right\rangle, G_{2}=\langle\beta\rangle$, $G_{3}=\left\langle\alpha^{2}\right\rangle$.

Notation 2.2. We will use the following notation for the rest of the paper:

$$
\begin{array}{rlrl}
a & =\sqrt{n}+\sqrt{n-1}, & \mu & =i \sqrt[4]{4 n(n-1)}, \\
m & =2 n-1, & \zeta=\frac{1+i}{\sqrt{2}}, \\
K_{0} & =\mathbb{Q}(a, \zeta \mu), & \\
E_{1} & =E_{2}=E: Y^{2} Z=X^{3}-X Z^{2}, & \\
E_{3} & =E_{(n)}: Y^{2} Z=X(X-Z)(X-n Z) . &
\end{array}
$$


Proposition 2.3. The degree 2 maps

$$
\begin{aligned}
& \psi_{1}: C_{n} \longrightarrow E \\
& (x, y, z) \longrightarrow\left(i\left(y^{2}-\zeta^{2} \mu^{2} x^{2}\right),(i-1)\left(z^{2}-m x^{2}\right),(y-\zeta \mu x)^{2}\right), \\
& \psi_{2}: C_{n} \longrightarrow E \\
& (x, y, z) \longrightarrow\left(i\left(y^{2}-\zeta^{2} \mu^{2} z^{2}\right),(i-1)\left(x^{2}-m z^{2}\right),(y-\zeta \mu z)^{2}\right), \\
& \psi_{n}: \quad C_{n} \quad \longrightarrow E_{(n)} \\
& (x, y, z) \longrightarrow\left(2\left(a^{2}+1\right)(z-a x)(a z-x),\left(a^{4}-1\right) y^{2}, 4 a(z-a x)^{2}\right)
\end{aligned}
$$

are the quotient maps $C_{n} \longrightarrow C_{n} / G_{1}, C_{n} \longrightarrow C_{n} / G_{2}, C_{n} \longrightarrow C_{n} / G_{3}$, respectively. They induce an isogeny of degree 8

$$
\Psi: J\left(C_{n}\right) \longrightarrow E \times E \times E_{(n)},
$$

defined over the field $K_{0}$.

This isogeny will be the key of our calculations, because it relates the type of reduction of the curve $C_{n}$ with that of the elliptic curves $E, E_{(n)}$, which is easy to determine.

An automorphism $\varphi$ of $C_{n}$ which commutes with $G_{i}$ induces an automorphism $\varphi^{E_{i}}$ of the elliptic curve $E_{i}$. Let us compute these induced automorphisms.

Proposition 2.4. The automorphisms of $C_{n}$ induce the following automorphisms of $E$ through the quotient map $\psi_{2}$ :

$$
\begin{aligned}
& \varphi_{00}^{E}=\varphi_{12}^{E}=\operatorname{Id}_{E}, \\
& \varphi_{01}^{E}=\varphi_{13}^{E}=[-i]_{E}+(1,0,1), \\
& \varphi_{02}^{E}=\varphi_{10}^{E}=[-1]_{E}+(0,0,1), \\
& \varphi_{03}^{E}=\varphi_{11}^{E}=[i]_{E}+(-1,0,1) .
\end{aligned}
$$

Proof. It is enough to consider the affine part $Z=1$. Put $x=X / Z$, $y=Y / Z$, so that

$$
(u, v):=\psi_{2}(x, y)=\left(i \frac{y+\zeta \mu}{y-\zeta \mu},(i-1) \frac{x^{2}-m}{(y-\zeta \mu)^{2}}\right) .
$$

After some algebraic manipulation we obtain that

$$
\varphi_{01}^{E}(u, v)=\left(\frac{u-1}{u+1},-\frac{2 i v}{(u+1)^{2}}\right) .
$$

We will now identify this map. Let us consider the associated map $f(Q)=$ $\varphi_{01}^{E}(Q)+P_{1}$, where $P_{1}=\varphi_{01}^{E}\left(O_{E}\right)=(1,0)\left(O_{E}\right.$ denotes the point at infinity of $E$ ). We may use the addition formulas for $E$ to calculate the equations which define $f$. We find that:

$$
f(u, v)=(-u, i v) .
$$


Thus, the map $f$ is multiplication by $-i$ on $E$, and hence it follows that $\varphi_{01}^{E}=[-i]_{E}+P_{1}$. The rest of the cases are solved in a similar way. Note that they can be grouped in pairs of the form $\varphi, \varphi \circ \beta$.

The same kind of calculations yields:

Proposition 2.5. The automorphisms of $C_{n}$ induce the following automorphisms of $E_{(n)}$ through the quotient map $\psi_{3}$ :

$$
\begin{array}{ll}
\varphi_{00}^{E_{(n)}}=\varphi_{02}^{E_{(n)}}=\operatorname{Id}_{E_{(n)}}, & \varphi_{01}^{E_{(n)}}=\varphi_{03}^{E_{(n)}}=[-1]_{E_{(n)}}, \\
\varphi_{11}^{E_{(n)}}=\varphi_{13}^{E_{(n)}}=\operatorname{Id}_{E_{(n)}}+P_{n}, & \varphi_{10}^{E_{(n)}}=\varphi_{12}^{E_{(n)}}=[-1]_{E_{(n)}}+P_{n}, \\
\varphi_{21}^{E_{(n)}}=\varphi_{23}^{E_{(n)}}=\operatorname{Id}_{E_{(n)}}+P_{0}, & \varphi_{20}^{E_{(n)}}=\varphi_{22}^{E_{(n)}}=[-1]_{E_{(n)}}+P_{0}, \\
\varphi_{31}^{E_{(n)}}=\varphi_{33}^{E_{(n)}}=\operatorname{Id}_{E_{(n)}}+P_{1}, & \varphi_{30}^{E_{(n)}}=\varphi_{32}^{E_{(n)}}=[-1]_{E_{(n)}}+P_{1},
\end{array}
$$

where $P_{t}=(t, 0,1)$.

\section{Reduction of the curves $C_{n}$.}

We now begin the arithmetical study of the curves $C_{n}$. For the definitions and basic results concerning reduction of curves, we refer to [Ds81].

For $n \not \equiv 0,1\left(\bmod 2^{5}\right)$, let us denote by $\mathcal{X}_{n}$ the arithmetic surface

$$
\operatorname{Proj}\left(\mathbb{Z}[X, Y, Z] /\left(Y^{4}-X^{4}+(4 n-2) X^{2} Z^{2}-Z^{4}\right)\right) \text {. }
$$

This surface is a model over $\mathbb{Z}$ of the curve $C_{n}$. Hence, the generic fibre of $\mathcal{X}_{n}$ is smooth, but $\mathcal{X}_{n}$ has singularities in some special fibres.

Proposition 3.1. The arithmetic surface $\mathcal{X}_{n}$ has good reduction outside the primes dividing $n(n-1)$.

Proof. The special fibre of $\mathcal{X}_{n}$ in a prime $p$ is given by the reduction $\bmod p$ of the equation $Y^{4}=X^{4}-(4 n-2) X^{2} Z^{2}+Z^{4}$. This reduction is singular only at the double roots of the polynomial $X^{4}-(4 n-2) X^{2} Z^{2}+Z^{4}$. The discriminant of this polynomial is $2^{12} n^{2}(n-1)^{2}$.

We must centre our attention on the primes dividing $n(n-1)$. It is wellknown that there exists a stable model of $C_{n}$ over the ring of integers of a certain number field $K_{n}$. We will determine this field and the stable model of $C_{n}$, taking into account the isogeny $\Psi$. We introduce some more notations:

Notation 3.2. Let $\alpha=\sqrt[3]{18-6 \sqrt{3}}$, and let $\alpha_{n}$ be a root of the equation

$$
t^{3}\left(t^{3}-24\right)^{3}-2^{8} \frac{\left(n^{2}-n+1\right)^{3}}{n^{2}(n-1)^{2}}\left(t^{3}-27\right)=0 .
$$

We put $K_{n}=K_{0}\left(\alpha, \alpha_{n}\right)$, and we denote by $\mathcal{O}_{n}$ the ring of integers of $K_{n}$, and write $S_{n}=\operatorname{Spec}\left(\mathcal{O}_{n}\right)$. 
We enumerate the reduction properties of the elliptic curves $E, E_{(n)}$ in the following two propositions. Note that the algebraic numbers $\alpha, \alpha_{n}$ allow to define the Deuring normal form of the curves (cf. [Si85]). The technical condition $n \not \equiv 0,1\left(\bmod 2^{5}\right)$ is necessary to assure the potentially good reduction at $p=2$ of the curve $E_{(n)}$.

\section{Proposition 3.3.}

a) The elliptic curve $E: Y^{2}=X^{3}-X$ has good reduction outside $p=2$, where it has potentially good reduction.

b) The curve $E$ has good reduction everywhere over the field $\mathbb{Q}(\alpha)$. Its Deuring normal form $Y^{2}+\alpha X Y+Y=X^{3}$ has good reduction at the primes dividing 2.

c) The curve $E$ is supersingular for a prime $p$ if and only if $p=2$ or $p \equiv 3(\bmod 4)$.

\section{Proposition 3.4.}

a) The $j$-invariant of the curve $E_{(n)}$ is

$$
j_{n}=2^{8} \frac{\left(n^{2}-n+1\right)^{3}}{n^{2}(n-1)^{2}},
$$

and hence the curve has good reduction outside the primes dividing $n(n-1)$.

b) If $p \mid n(n-1)$ is an odd prime, $E_{(n)}$ has multiplicative reduction at $p$.

c) The curve $E_{(n)}$ has potentially good reduction at $p=2$. Over the field $\mathbb{Q}\left(\alpha_{n}\right)$, the curve $E_{(n)}$ has good reduction at the primes dividing 2. Its Deuring normal form $Y^{2}+\alpha_{n} X Y+Y=X^{3}$ has good reduction at these primes.

d) The curve $E_{(n)}$ is supersingular at $p=2$. It is supersingular at $p=3$ if and only if $n \equiv 2(\bmod 3)$. If $E_{(n)}$ is supersingular at a prime $p>3$ then $p \equiv 3(\bmod 4)$.

The assertions concerning the supersingularity of $E$ and $E_{n}$ follow from the characterization of supersingular elliptic curves, and from the fact that $E_{n}\left(\mathbb{F}_{p}\right)$ has an evident subgroup of order 4 whenever $p>2$.

Theorem 3.5. Let $J_{n}=\operatorname{Pic}_{\mathcal{X}_{n} / K_{n}}^{0}$ be the Jacobian of $\mathcal{X}_{n / K_{n}}=C_{n}$, and let $\mathcal{N}$ be its Néron model over $\mathcal{O}_{n}$.

a) The curve $C_{n}$ has a stable model $\mathcal{X}_{n}^{\text {st }}$ and a semistable minimal regular model $\mathcal{X}_{n}^{\text {reg }}$ over $\mathcal{O}_{n}$.

b) At the primes $\mathfrak{p}$ in $\mathcal{O}_{n}$ which divide $2, \mathcal{N}$ has abelian reduction.

c) At the odd primes $\mathfrak{p}$ in $\mathcal{O}_{n}$ which divide $n(n-1), \mathcal{N}$ has semi-abelian reduction, and its toric part has dimension 1.

d) At any prime $\mathfrak{p}$ in $\mathcal{O}_{n}$ we have canonical isomorphisms over the residual field $k_{\mathfrak{p}}$ :

$$
\operatorname{Pic}_{\mathcal{X}_{n}^{\text {st }} / k_{\mathfrak{p}}}^{0} \simeq \operatorname{Pic}_{\mathcal{X}_{n}^{\text {reg }} / k_{\mathfrak{p}}}^{0} \simeq \mathcal{N}_{\mathfrak{p}}^{0}
$$


Proof. The elliptic curves $E, E_{(n)}$ have semi-stable reduction over $K_{n}$, so that the isogeny $\Psi$ guarantees that $J_{n}$ also has semi-stable reduction over $K_{n}$, by the Néron-Ogg-Safarevic criterion ([Se-Ta68]). But this is equivalent to the existence of a stable model $\mathcal{X}_{n}^{\text {st }}$ for $C_{n}$ over $K_{n}$ (cf. [De-Mu69]). Blowing up the singular points of $\mathcal{X}_{n}^{\text {st }}$ we obtain a semistable minimal regular model.

We can extend the isogeny $\Psi$ to the Néron models of $J_{n}, E, E_{(n)}$, and again the criterion of Néron-Ogg-Safarevic translates Propositions 3.3 and 3.4 into Parts b) and c).

Finally, the second isomorphism in Part d) is well-known (cf. [BLR90]), while the first isomorphism is given by (the reduction of) the map from $\mathcal{X}_{n}^{\text {reg }}$ onto $\mathcal{X}_{n}^{\text {st }}$ which blows down the rational components with self-intersection -2 in the special fibre.

\section{Height of the curves $C_{n}$.}

We are already in position to calculate the modular height of the curves $C_{n}$. For the definition of the modular height and the remaining basic concepts of Arakelov geometry, we refer to [MB85] or [La88].

The modular height of abelian varieties has a good behaviour with respect to isogenies: Raynaud proved the following result:

Proposition 4.1 ([Ra85], Cor. 2.1.4). Let $A_{K}, B_{K}$ be two abelian varieties over a number field $K$, and let $\mathcal{A}, \mathcal{B}$ be their Néron models over $\mathcal{O}_{K}$. If there exists an isogeny $\phi: A_{K} \longrightarrow B_{K}$ of degree $p^{e}$, with $p$ a prime, then:

$$
h(\mathcal{A})=h(\mathcal{B})+k \log p,
$$

for some $k \in \mathbb{Z},|k| \leq e / 2$.

Being clear that the height of a product of abelian schemes is the sum of the heights of the factors, we will have a good approximation to the height of the curves $C_{n}$ once we have the height of the elliptic curves $E, E_{(n)}$. The computation of the height of an elliptic curve amounts to the computation of its period lattice and of its reduction, following Tate's algorithm. We obtain:

Proposition 4.2. The height of the elliptic curve $E_{(n)}$ is given by

$$
h\left(E_{(n)}\right)=\frac{1}{6} \log n(n-1)-\frac{1}{6} v_{2}(n(n-1)) \log 2-\frac{1}{12} \log \left(\left|\Delta\left(\tau_{n}\right)\right|\left(\operatorname{Im} \tau_{n}\right)^{6}\right),
$$

where

$$
\tau_{n}=i K(\sqrt{1-1 / n}) / K(1 / \sqrt{n})
$$

is the fundamental period of $E_{(n)}, K()$ denotes the complete elliptic integral of the first kind, $v_{2}$ is the 2 -adic valuation and $\Delta$ is the discriminant modular form. 
Remark. For $n=2$ this formula gives $h(E)=\log \frac{\Gamma(3 / 4) \sqrt{2}}{\Gamma(1 / 4) \sqrt{\pi}}$, as in [De85].

The modular height of a curve is that of the Néron model of its Jacobian. Combining Propositions 4.1 and 4.2 we arrive at:

Theorem 4.3. The height of the curve $C_{n}$ is given by

$$
\begin{aligned}
h\left(C_{n}\right)= & 2 \log \frac{\Gamma(3 / 4) \sqrt{2}}{\Gamma(1 / 4) \sqrt{\pi}}+\frac{1}{6} \log \frac{n(n-1)}{2^{v_{2}(n(n-1))}} \\
& -\frac{1}{12} \log \left(\left|\Delta\left(\tau_{n}\right)\right|\left(\operatorname{Im} \tau_{n}\right)^{6}\right)+k \log 2,
\end{aligned}
$$

for a certain $k \in\{0, \pm 1\}$ (which depends on $n)$.

We can draw a graphic of the height $h\left(C_{n}\right)$ as a function of $n$, where its logarithmic behaviour when $n$ approaches the singular curves of the family $(n=1, n \rightarrow \infty)$ will become evident:

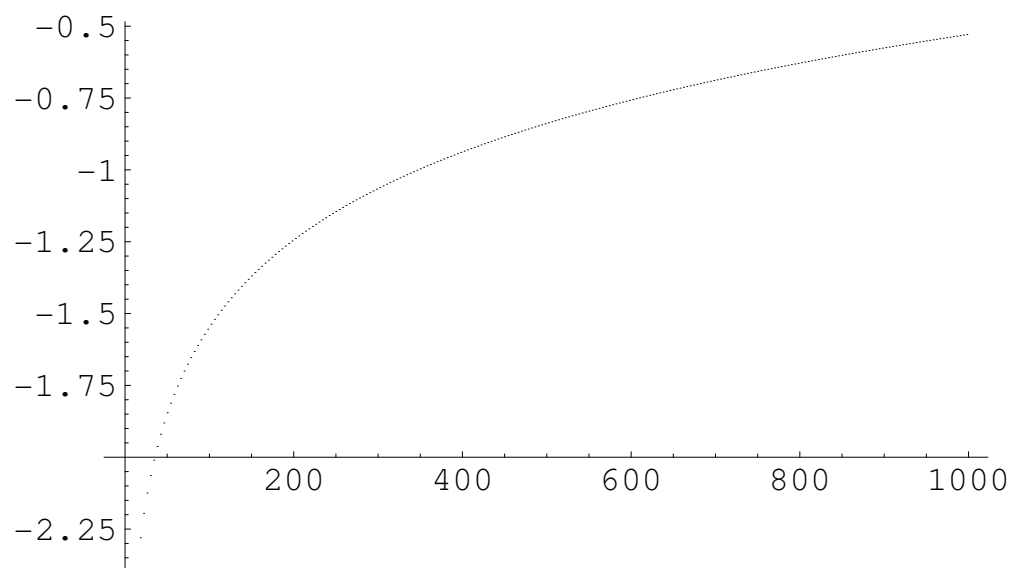

Figure 1.

5. The stable model of the curves $C_{n}$ over odd primes.

Let $p \mid n(n-1)$ be an odd prime, and let $\mathfrak{p}$ be a prime divisor of $p$ in $\mathcal{O}_{n}$. We will now determine the fibre over $\mathfrak{p}$ of $\mathcal{X}_{n}^{\text {st }}$.

Lemma 5.1. The irreducible components of $\mathcal{X}_{n / k_{\mathfrak{p}}}^{\text {st }}$ are smooth.

Proof. If we reduce the equation of $C_{n} \bmod p$

$$
\begin{aligned}
Y^{4}=X^{4}-(4 n-2) X^{2} Z^{2}+Z^{4} & =\left(X^{2}+Z^{2}\right)^{2}-4 n X^{2} Z^{2} \\
& =\left(X^{2}-Z^{2}\right)^{2}-4(n-1) X^{2} Z^{2},
\end{aligned}
$$


we have that the fibre at $\mathfrak{p}$ has equation $\left(Y^{2}-X^{2}-Z^{2}\right)\left(Y^{2}+X^{2}+Z^{2}\right)=0$ if $p$ divides $n$, and $\left(Y^{2}-X^{2}+Z^{2}\right)\left(Y^{2}+X^{2}-Z^{2}\right)=0$ if $p$ divides $n-1$. In both cases, the fibre is reduced and its components are smooth and rational. Thus, we can obtain a semi-stable minimal regular model for $C_{n}$ blowingup, normalizing and blowing down exceptional components. None of these operations introduces singular components. The stable model is obtained from the minimal regular model contracting the rational components with self-intersection -2 , but again this does not introduce singularities ([Li69]).

Lemma 5.2. $\mathcal{X}_{n / k_{\mathfrak{p}}}^{\text {st }}$ is not irreducible.

Proof. The irreducibility of $\mathcal{X}_{n / k_{\mathfrak{p}}}^{s t}$ would imply that it is a nonsingular curve of genus 3, and then $\operatorname{Pic}_{\mathcal{X}_{n}^{s t} / k_{\mathfrak{p}}}^{0}$ would be an abelian variety of dimension 3 , contradicting Theorem 3.5.

These results drastically reduce the number of possible configurations for $\mathcal{X}_{n / k_{\mathfrak{p}}}^{s t}$. The dimension of the toric part of $\operatorname{Pic}_{\mathcal{X}_{n}^{\text {st }} / k_{\mathfrak{p}}}^{0}$ being 1 (Theorem 3.5), the geometric configuration of the fibre must form exactly one cycle (cf. [BLR90], pp. 245-249).

Suppose that $\mathcal{X}_{n / k_{\mathfrak{p}}}^{s t}$ has a component of genus 2 . Then, the other components should be rational, and they should form a cycle. But this is impossible, since the rational components must intersect the other components at least at three points. Hence, the components of $\mathcal{X}_{n / k_{\mathfrak{p}}}^{s t}$ must be rational or elliptic. It remains only one possibility: $\mathcal{X}_{n / k_{\mathfrak{p}}}^{s t}$ must have two elliptic components, intersecting at two points:

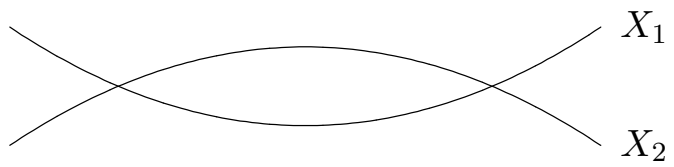

Figure 2.

Theorem 5.3. The special fibre of $\mathcal{X}_{n}^{\text {st }}$ at an odd prime $\mathfrak{p}$ in $\mathcal{O}_{n}$ dividing $n(n-1)$ has two elliptic components $X_{1, \mathfrak{p}}, X_{2, \mathfrak{p}}$, intersecting at two different points.

\section{Automorphisms of $\mathcal{X}_{n}^{s t}$.}

The automorphisms of $C_{n}$ can be extended to automorphisms of the stable model $\mathcal{X}_{n}^{\text {st }}($ cf. $[$ Ra90]), which we will denote by the same letters. The 
quotients $\mathcal{E}_{i}^{0}:=\mathcal{X}_{n}^{s t} / G_{i}$ exist and are semi-stable curves (cf. [Ra90]). The generic fibre of $\mathcal{E}_{i}^{0}$ is the elliptic curve $E_{i}=E$ for $i=1,2$, and the elliptic curve $E_{3}=E_{(n)}$ for $i=3$.

An automorphism $\varphi$ of $\mathcal{X}_{n}^{\text {st }}$ which commutes with $G_{i}$ induces an automorphism $\varphi^{\mathcal{E}_{i}^{0}}$ of $\mathcal{E}_{i}^{0}$, leaving the smooth part invariant, and hence determines an automorphism $\varphi^{\mathcal{E}_{i}}$ of the Néron model $\mathcal{E}_{i}$ of the elliptic curve $E_{i}$. We will denote by $\varphi^{E_{i / k_{\mathfrak{p}}}}$ the reduction of this automorphism $\bmod$ a prime $\mathfrak{p}$, i.e., the automorphism induced by $\varphi$ in the special fibre of $\mathcal{E}_{i}$. We remark that at good reduction primes the special fibres of $\mathcal{E}_{i}$ and $\mathcal{E}_{i}^{0}$ are the same, and we have a canonical isomorphism between them.

When $E_{i}$ has good reduction at $\mathfrak{p}$, we can read the maps $\varphi^{E_{i / k_{\mathfrak{p}}}}$ from the maps $\varphi^{E_{i}}$ calculated in Propositions 2.4 and 2.5, thanks to the universal property of the Néron model. We also have to control the reduction of the points appearing on those propositions, i.e., we have to control the supersingular character of $E_{i}$ at $\mathfrak{p}$, which we know from Propositions 3.3 and 3.4. We obtain:

Theorem 6.1. Let $\mathfrak{p}$ be a prime in $\mathcal{O}_{n}$ dividing 2 .

a) The automorphisms of $C_{n}$ induce the following automorphisms in the special fibre $E_{/ k_{\mathfrak{p}}}$ of $\mathcal{E}$ at $\mathfrak{p}$ :

$$
\begin{array}{ll}
\varphi_{00}^{E_{/ k_{\mathfrak{p}}}}=\varphi_{10}^{E_{/ k_{\mathfrak{p}}}}=\operatorname{Id}_{E_{/ k_{\mathfrak{p}}},}, & \varphi_{02}^{E_{/ k_{\mathfrak{p}}}}=\varphi_{12}^{E_{/ k_{\mathfrak{p}}}}=[-1]_{E_{/ k_{\mathfrak{p}}}}, \\
\varphi_{01}^{E_{/ k_{\mathfrak{p}}}}=\varphi_{11}^{E_{/ k_{\mathfrak{p}}}}=[-i]_{E_{/ k_{\mathfrak{p}}}}, & \varphi_{03}^{E_{/ k_{\mathfrak{p}}}}=\varphi_{13}^{E_{/ k_{\mathfrak{p}}}}=[i]_{E_{/ k_{\mathfrak{p}}}} .
\end{array}
$$

b) The automorphisms of $C_{n}$ induce the following automorphisms in the special fibre $E_{(n) / k_{\mathfrak{p}}}$ of $\mathcal{E}_{n}$ at $\mathfrak{p}$ :

$$
\begin{aligned}
& \varphi_{00}^{E_{(n) / k_{\mathfrak{p}}}}=\varphi_{02}^{E_{(n) / k_{\mathfrak{p}}}}=\varphi_{11}^{E_{(n) / k_{\mathfrak{p}}}}=\varphi_{13}^{E_{(n) / k_{\mathfrak{p}}}}= \\
& \varphi_{21}^{E_{(n) / k_{\mathfrak{p}}}}=\varphi_{23}^{E_{(n) / k_{\mathfrak{p}}}}=\varphi_{31}^{E_{(n) / k_{\mathfrak{p}}}}=\varphi_{33}^{E_{(n) / k_{\mathfrak{p}}}}=\operatorname{Id}_{E_{(n) / k_{\mathfrak{p}}}}, \\
& \varphi_{01}^{E_{(n) / k_{\mathfrak{p}}}}=\varphi_{03}^{E_{(n) / k_{\mathfrak{p}}}}=\varphi_{10}^{E_{(n) / k_{\mathfrak{p}}}}=\varphi_{12}^{E_{(n) / k_{\mathfrak{p}}}}= \\
& \varphi_{20}^{E_{(n) / k_{\mathfrak{p}}}}=\varphi_{22}^{E_{(n) / k_{\mathfrak{p}}}}=\varphi_{30}^{E_{(n) / k_{\mathfrak{p}}}}=\varphi_{32}^{E_{(n) / k_{\mathfrak{p}}}}=[-1]_{E_{(n) / k_{\mathfrak{p}}}}
\end{aligned}
$$

Note that this implies that the points in $E_{/ k_{\mathfrak{p}}}$ fixed by the induced automorphisms must be 2 -torsion points. The same kind of reasoning proves that, at the odd primes, the points in the special fibre of $E_{/ k_{\mathfrak{p}}}$ fixed by the induced automorphisms must be 4 torsion points, a fact that will be used in Proposition 9.2. 


\section{The stable model of the curves $C_{n}$ over $p=2$.}

We now determine the fibre of $\mathcal{X}_{n}^{\text {st }}$ over a prime $\mathfrak{p}$ of $\mathcal{O}_{n}$ dividing 2 . In analogy with Lemmas 5.1 and 5.2, we have:

\section{Lemma 7.1.}

a) $\operatorname{Pic}_{\mathcal{X}_{n}^{\text {st }} / k_{\mathfrak{p}}}^{0}$ is an abelian variety of dimension 3 .

b) The geometric configuration of $\mathcal{X}_{n / k_{\mathfrak{p}}}^{\text {st }}$ contains no cycle.

c) The irreducible components of $\mathcal{X}_{n / k_{\mathfrak{p}}}^{\text {st }}$ are smooth.

Proof. The first assertion follows from the isogeny $\Psi$. Parts b) and c) are consequence of a) by [BLR90], Cor. 9.12.

The study of the special fibre of $\mathcal{X}_{n / k_{\mathfrak{p}}}^{s t}$ is not so easy in this case, since the dimension of the Jacobian does not provide further information. We are now obliged to use the results obtained in previous section.

Proposition 7.2. $\mathcal{X}_{n / k_{\mathfrak{p}}}^{s t}$ is not irreducible.

Proof. The reduction of the quotient maps $\psi_{i}: \mathcal{X}_{n / k_{\mathfrak{p}}}^{s t} \longrightarrow \mathcal{E}_{i}^{0}$ yields an isogeny of degree 8

$$
\Psi_{\mathfrak{p}}: \operatorname{Pic}_{\mathcal{X}_{n / k_{\mathfrak{p}}}^{s t}}^{0} \longrightarrow E_{/ k_{\mathfrak{p}}} \times E_{/ k_{\mathfrak{p}}} \times E_{(n) / k_{\mathfrak{p}}} .
$$

As $\operatorname{Pic}_{\mathcal{X}_{n / k \mathfrak{p}}^{s t}}^{0}$ is an abelian variety, we can consider the dual isogeny $\Phi_{p}$ of $\Psi_{\mathfrak{p}}$. The Hasse-Witt invariant of the elliptic curves $E, E_{(n)}$ is 0 , since its $j$ invariant is 0 . Hence, their product admits no separable isogenies of degree a power of 2. Thus, the composition $\Psi_{\mathfrak{p}} \circ \Phi_{\mathfrak{p}}=[2]$ must be purely inseparable, which implies that also $\Psi_{\mathfrak{p}}$ is purely inseparable.

Suppose that $\mathcal{X}_{n / k_{\mathfrak{p}}}^{s t}$ is irreducible. It should be smooth, since its Jacobian has dimension 3. As the maps $\psi$ have degree 2, they must be separable or purely inseparable. In the last case, $\mathcal{X}_{n / k_{\mathfrak{p}}}^{s t}$ would be isomorphic to the elliptic curve $E_{/ k_{\mathfrak{p}}}$, which is not possible. Hence, the three maps $\psi_{1}, \psi_{2}, \psi_{3}$ should be separable. But the isogeny $\Psi_{\mathfrak{p}}$ that they induce would be also separable (this can be seen in terms of differential forms), and we have seen that this is not the case.

The reduction of the maps $\psi_{i}$ being exhaustive, we must have non-rational irreducible components $X_{1}, X_{2}, X_{(n)}$ such that $\left.\psi_{i}\right|_{X_{i}}: X_{i} \longrightarrow E_{i / k_{\mathfrak{p}}}$ is a degree 2 map. A priori, we do not know whether these components are really different or they coincide. In any case, we know that $\mathcal{X}_{n / k_{\mathfrak{p}}}^{s t}$ cannot have more than three non-rational components, since it has genus 3 .

Let $X_{i}$ be one of the non-rational components of $\mathcal{X}_{n / k_{\mathfrak{p}}}^{s t}$. If $\psi_{i}\left(X_{i}\right)$ is not a point, then $\left.\psi_{i}\right|_{X_{i}}$ must be purely inseparable (otherwise the isogeny $\Psi_{\mathfrak{p}}$ 
would not be purely inseparable), i.e., it must be a degree 2 Frobenius map. In particular, $X_{i}$ must have genus 1 .

Let us consider the reduction of the automorphism $\varphi_{01}$ over $\mathcal{X}_{n / k_{\mathfrak{p}}}^{s t}$, and its action on $E_{1 / k_{\mathfrak{p}}}$ and $E_{(n) / k_{\mathfrak{p}}}$ :

$$
\varphi_{01}^{E_{1 / k_{\mathfrak{p}}}}=[-i]_{E_{1 / k_{\mathfrak{p}}}}, \quad \varphi_{01}^{E_{(n) / k_{\mathfrak{p}}}}=[-1]_{E_{(n) / k_{\mathfrak{p}}}} .
$$

As $\psi_{1} \mid X_{1}$ and $\psi_{3} \mid X_{n}$ are bijective maps, we see that $\varphi_{01}\left(X_{1}\right)=X_{1}, \varphi_{01}\left(X_{(n)}\right)$ $=X_{(n)}$. Moreover,

$$
\psi_{01} \mid X_{1}=\psi_{1 / k_{\mathfrak{p}}}^{-1} \circ \varphi_{01}^{E_{1 / k_{\mathfrak{p}}}} \psi_{1 / k_{\mathfrak{p}}}=\psi_{1 / k_{\mathfrak{p}}}^{-1} \circ[-i]_{E_{1 / k_{\mathfrak{p}}}} \psi_{1 / k_{\mathfrak{p}}},
$$

and hence $\psi_{01} \mid X_{1}=[-1]_{X_{1}}$. We can see in the same way that $\psi_{01} \mid X_{(n)}=$ $\operatorname{Id}_{X_{(n)}}$. This fact ensures that $X_{1} \neq X_{(n)}$. We can argue similarly to prove that $X_{2} \neq X_{(n)}$ and $X_{1} \neq X_{2}$. At this moment we have seen that the special fibre $\mathcal{X}_{n / k_{\mathfrak{p}}}^{s t}$ has exactly three elliptic components, and the rest of the components must be rational. We know that $\psi_{2}=\psi_{1} \circ \gamma$, so that $X_{2}=\gamma\left(X_{1}\right)$, and this forces $\gamma\left(X_{(n)}\right)=X_{(n)}$. Moreover, $X_{1} \cap X_{(n)} \neq \emptyset$ if and only if $X_{2} \cap X_{(n)} \neq \emptyset$. Gathering all these restrictions on the special fibre $\mathcal{X}_{n / k_{\mathfrak{p}}}^{s t}$, only two possibilities remain:

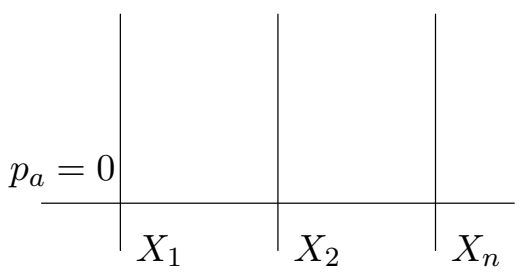

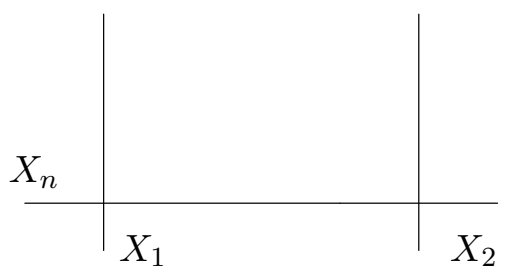

Figure 3.

Let us denote by $O_{i}$ the neutral point of the group law on the elliptic component $X_{i}$.

Lemma 7.3. The components $X_{1}, X_{(n)}$ can intersect only at their neutral point. The same is true for the components $X_{2}, X_{(n)}$.

Proof. Let $P \in X_{1} \cap X_{(n)}$. We know that $\varphi_{12} \mid X_{(n)}=\operatorname{Id}_{\left.X_{(n)}\right)}$, so that $\varphi_{12}(P)=P$. On the other hand, $\varphi_{12} \mid X_{1}=[-1]_{X_{1}}$ implies $\varphi_{12}(P)=-P$, where we denote by $-P$ the opposite of $P$ with respect to the group law on $X_{1}$. The component $X_{1}$ is isomorphic to the elliptic curve $E_{/ k_{\mathfrak{p}}}$, which is supersingular, so that there are no 2 -torsion points on $X_{1}$, that is, we must have $P=O_{1}$. Using the automorphism $\varphi_{01}$ we see that we must also have $P=O_{(n)}$. The second assertion is proved using the automorphism $\gamma$. 
The lemma excludes the second option in Figure 3. We have finally reached:

Theorem 7.4. The special fibre of the stable model of $C_{n}$ at a prime $\mathfrak{p}$ in $\mathcal{O}_{n}$ dividing 2 consists of three elliptic components $X_{1, \mathfrak{p}}, X_{2, \mathfrak{p}}, X_{3, \mathfrak{p}}$, which intersect a rational component $X_{0, \mathfrak{p}}$ at three different points. The map $\psi_{i}$ : $\mathcal{X}_{n / k_{\mathfrak{p}}}^{s t} \longrightarrow E_{/ k_{\mathfrak{p}}}$ restricted to the component $X_{i, \mathfrak{p}}$ is the Frobenius map, and contracts the other components.

\section{A lower bound for Arakelov self-intersection.}

We now begin the study of the self-intersection of the Arakelov dualizing sheaf of the arithmetic surfaces given by the curves $C_{n}$. For the definitions, we refer again to [MB85] or [La88].

In this section we use a result of Moriwaki to precise a lower bound for the self-intersection of the canonical sheaf of the curves $C_{n}$.

Proposition 8.1 ([Mo96]). Let $\mathcal{X} \longrightarrow S=\operatorname{Spec}\left(\mathcal{O}_{K}\right)$ a stable arithmetic surface of genus $g \geq 2$. Let $\mathfrak{p}_{1}, \ldots, \mathfrak{p}_{t}$ be the primes in $\mathcal{O}_{K}$ where the fibres of $\mathcal{X}$ are reducible. We have that

$$
\left(\bar{\omega}_{\mathcal{X} / S}, \bar{\omega}_{\mathcal{X} / S}\right)_{\mathrm{Ar}} \geq \frac{1}{6(g-1)} \sum_{i=1}^{t} \log N_{K / \mathbb{Q}}\left(\mathfrak{p}_{i}\right) .
$$

We apply this result to the arithmetic surface given by the curve $C_{n}$ to obtain:

Proposition 8.2. For the stable model $\mathcal{X}_{n}^{\text {st }} \longrightarrow S_{n}$ of the curve $C_{n}$ over the ring of integers of the field $K_{n}$, we have:

$$
\left(\bar{\omega}_{\mathcal{X}_{n}^{\text {st }} / S_{n}}, \bar{\omega}_{\mathcal{X}_{n}^{\text {st }} / S_{n}}\right)_{\mathrm{Ar}} \geq \frac{1}{12} \log \prod_{\mathfrak{p} \in S_{n}, \mathfrak{p} \mid n(n-1)} N_{K_{n} / \mathbb{Q}}(\mathfrak{p}) .
$$

The normalized Arakelov self-intersection of a curve $C_{K}$ defined over a number field $K$ is

$$
e\left(C_{K}\right):=\frac{1}{[L: K]}\left(\omega_{\mathcal{C}^{s t}}, \omega_{\mathcal{C}^{s t}}\right) \mathrm{Ar},
$$

where $L / K$ is an extension over which $C_{K}$ has a stable model $\mathcal{C}^{s t}$. The curves $C_{n}$ provide examples of curves with normalized Arakelov self-intersection as large as desired:

Theorem 8.3. For any $H>0$, there exist infinitely many curves $C_{\mathbb{Q}} d e-$ fined over $\mathbb{Q}$ such that $e\left(C_{\mathbb{Q}}\right)>H$.

Proof. We know from Sections 5 and 7 that the primes where the stable model of the curve $C_{n}$ has reducible fibres are those dividing $n(n-1)$. 
Hence, the curves $C_{n}$ satisfy

$$
e\left(C_{n}\right) \geq \frac{1}{12\left[K_{n}: \mathbb{Q}\right]} \log \prod_{p \mid n(n-1)} p .
$$

The degree of the extension $K_{n} / \mathbb{Q}$ is always less than or equal to 1152 , while we can take infinitely many values of $n$ which make the product $\prod_{p \mid n(n-1)} p$ as large as desired.

\section{Canonical divisors.}

We will ultimately give an explicit formula for the self-intersection of the Arakelov dualizing sheaf of the arithmetic surfaces given by the curves $C_{n}$. The purpose of this section is the determination of two canonical divisors on the minimal model of these curves. We shall need to make a base extension to determine such divisors.

Let us consider the following points on the (affine part of the) curve $C_{n}(\overline{\mathbb{Q}}):$

$$
\begin{aligned}
& P_{1}=\left(\sqrt{2 n-2 u \sqrt{n(n-1)}-1}, \frac{\sqrt[4]{12}-\sqrt{3}-1}{2} \zeta \mu\right), \\
& P_{2}=\left(-\sqrt{2 n-2 u \sqrt{n(n-1)}-1}, \frac{\sqrt[4]{12}-\sqrt{3}-1}{2} \zeta \mu\right), \\
& P_{3}=\left(\sqrt{2 n+2 u \sqrt{n(n-1)}-1}, \frac{\sqrt[4]{12}-\sqrt{3}-1}{2} \zeta \mu\right), \\
& P_{4}=\left(-\sqrt{2 n+2 u \sqrt{n(n-1)}-1}, \frac{\sqrt[4]{12}-\sqrt{3}-1}{2} \zeta \mu\right),
\end{aligned}
$$

where $u=\sqrt{-6+4 \sqrt[4]{12}-4 \sqrt{3}+2 \sqrt[4]{108}}$. The images of these points through the map $\psi_{2}$ are two different nontrivial 3 -torsion points $R_{1}=\psi_{2}\left(P_{1}\right)=$ $\psi_{2}\left(P_{2}\right), R_{2}=-R_{1}=\psi_{2}\left(P_{3}\right)=\psi_{2}\left(P_{4}\right)$ of the elliptic curve $E$.

In the proof of Lemma 10.2, we shall need the following result:

Lemma 9.1. The pair of points $P_{1}, P_{2}$ is conjugate over $\mathbb{Q}$ to the pair of points $P_{3}, P_{4}$.

Proof. It is enough to see that the pairs are conjugate over the larger field $L=\mathbb{Q}(b \zeta \mu)$, where $b=\frac{\sqrt[4]{12}-\sqrt{3}-1}{2}$. This, in turn, reduces to proving that $u^{2} n(n-1)$ is not a square in $L$. Since $\mathbb{Q}(b)=\mathbb{Q}\left(b^{4}\right)=\mathbb{Q}(\sqrt[4]{12}) \subset L=$ $\mathbb{Q}(\sqrt[4]{12}, \sqrt[4]{-4 n(n-1)})$, we have $\zeta \mu \in L$, and $(\zeta \mu)^{4}=-4 n(n-1)$ is a square in $L$. Hence, we must check that $-u^{2}=b^{4}-1 \notin L^{* 2}$. Let $F:=\mathbb{Q}\left(\sqrt{b^{4}-1}\right)$; we want to see that $[F L: L]=2$, or equivalently, that $[F L: F]=4$, so that it suffices to prove that the polynomial $X^{4}-(\zeta \mu)^{4}=X^{4}+4 n(n-1)$ is irreducible over $F$. This can only happen if $-4 n(n-1)$ is a square or -4 
times a fourth power in $F$. The first possibility is excluded, since $F \subset \mathbb{R}$. So, it remains to prove that $H:=\mathbb{Q}(\sqrt[4]{n(n-1)})$ may not be embedded in $F$. Using PARI ([BBBCO99]), we see that the only quartic subfield of $F$ is $M=\mathbb{Q}(\sqrt[4]{12})$. If $H=M$ the discriminants of the polynomials $X^{4}-12$ and $X^{4}-n(n-1)$ should agree up to a square, i.e., $3 n(n-1)$ should be a rational square, which is not possible since we are assuming that $n \equiv 2$ $(\bmod 3)$.

Let $L_{n}$ be a finite extension of the field $K_{n}\left(P_{1}, P_{3}\right)$ satisfying the following condition: The prime ideals in $K_{n}\left(P_{1}, P_{3}\right)$ which divide $n(n-1)$ become principal in $L_{n}$ (the convenience of this condition will be clear later, just before Theorem 9.4). We will denote by $T_{n}$ the spectrum of the ring of integers $\mathcal{O}_{n}^{\prime}$ of $L_{n}$, and by $\ell_{\mathfrak{p}}$ the residual field of $L_{n}$ at a prime $\mathfrak{p}$ in $\mathcal{O}_{n}^{\prime}$. Since the stable model is stable under base extensions, the stable model $\mathcal{V}_{n}$ of $C_{n}$ over $T_{n}$ is the base change of $\mathcal{X}_{n}^{\text {st }}$ to $\mathcal{O}_{n}^{\prime}$. In particular, the configuration of the special fibres of $\mathcal{V}_{n}$ is exactly the same of the special fibres of $\mathcal{X}_{n}^{\text {st }}$ (so that we are going to use the same notation for them).

Proposition 9.2. Let $U$ be the smooth part of $\mathcal{V}_{n}$, and let $U_{1}$ be the complement of the bad fibres of $\mathcal{V}_{n}$.

a) The points $P_{1}, P_{2}, P_{3}, P_{4}$ extend to sections $M_{1}, M_{2}, M_{3}, M_{4}$ on $\mathcal{V}_{n}$ contained in $U$.

b) Let $D_{1}^{0}=M_{1}+M_{2}+M_{3}+M_{4}$. We have a canonical isomorphism:

$$
\omega_{\mathcal{V}_{n} / T_{n}}\left|U_{1} \simeq \mathcal{O}_{\mathcal{V}_{n} / T_{n}}\left(D_{1}^{0}\right)\right| U_{1} .
$$

Proof. a) The non-smooth points in $\mathcal{V}_{n}$ are the singular points of the fibres at primes of bad reduction. At an odd prime of bad reduction, any automorphism $\varphi^{2}$ leaves the singular points of the fibre invariant, so that the corresponding induced automorphism on $E$ leaves invariants the images of these singular points through $\psi_{2}$. Hence, these points must be 4 -torsion points (cf. remark at the end of Section 6). In the fibres over even primes, a similar argument shows that the singular points must be the neutral elements of the elliptic components. The reductions of the sections $M_{i}$ are 3 -torsion points on the special fibre, which can only be trivial at places of characteristic 3, (where $E$ is supersingular), but these are primes of good reduction, since $n \equiv 2(\bmod 3)$. Thus, the sections $M_{i}$ do not pass through the non-smooth points of $\mathcal{V}_{n}$.

b) On $U_{1}$ we have an isomorphism $\omega_{\mathcal{V}_{n} / T_{n}}\left|U_{1} \simeq \Omega_{\mathcal{V}_{n} / T_{n}}\right| U_{1}$, where $\Omega_{\mathcal{V}_{n} / T_{n}}$ denotes the sheaf of relative differential forms. On the generic fibre $C_{n}$ we have

$$
\Omega_{C_{n}} \simeq \mathcal{O}_{C_{n}}\left(P_{1}+P_{2}+P_{3}+P_{4}\right),
$$

since the genus of $C_{n}$ is 3 , every plane embedding of $C_{n}$ is canonical, and the points $P_{i}$ are collinear. This isomorphism extends to an isomorphism 
$\Omega_{\mathcal{V}_{n} / T_{n}}\left|U_{1} \simeq \mathcal{O}_{\mathcal{V}_{n} / T_{n}}\left(D_{1}^{0}\right)\right| U_{1}$, since the surface $\mathcal{V}_{n} \mid U_{1}$ is described by a global nonsingular plane quartic, and the line through the points $P_{i}$ is globally defined over $\mathbb{Z}$, since it is of the form $y=\lambda$, with $\lambda$ and algebraic integer.

In order to extend the above isomorphism to the whole surface $\mathcal{V}_{n}$, we must add some vertical components of the bad fibres to the divisor $D_{1}^{0}$. To control these extra components, we have to determine the intersection of $D_{1}^{0}$ with the bad fibres.

\section{Lemma 9.3.}

a) Let $\mathfrak{p}$ an odd prime in $\mathcal{O}_{n}^{\prime}$ dividing $n(n-1)$. The divisor $D_{1}^{0}$ intersects each of the two components of $\mathcal{V}_{n, \mathfrak{p}}$ at two points (which may coincide).

b) Let $\mathfrak{p}$ a prime in $\mathcal{O}_{n}^{\prime}$ dividing 2 . The divisor $D_{1}^{0}$ intersects $X_{2, \mathfrak{p}}$ at two points with multiplicity 2 , and does not cut any other component of the fibre.

Proof. a) From $\varphi_{12}\left(P_{1}\right)=P_{2}$, we deduce that $\varphi_{12}\left(M_{1}\right)=M_{2}$. Moreover, $\varphi_{12}$ permutes $X_{1, \mathfrak{p}}$ and $X_{2, \mathfrak{p}}$, because $\psi_{1}=\psi_{2} \circ \varphi_{12}$. Thus, $M_{1}, M_{2}$ must intersect $\mathcal{V}_{n, \mathfrak{p}}$ at different components. The same is true for $M_{3}, M_{4}$.

b) The reduced map $\psi_{2, \mathfrak{p}} \mid X_{2, \mathfrak{p}}$ is a Frobenius map of degree 2, so that is totally ramified. The images of the reduction of the sections $M_{i}$ are two different 3-torsion points in the special fibre of $E$, and thus the $M_{i}$ must pass through two points on $X_{2, \mathfrak{p}}$.

From now on, we will work on the minimal regular model $\mathcal{V}_{n}^{\prime}$ of the curves $C_{n}$. It may be obtained blowing up the singularities of the stable model. Let us introduce some notation to describe the bad fibres of $\mathcal{V}_{n}^{\prime}$.

At a prime $\mathfrak{p}$ in $\mathcal{O}_{n}^{\prime}$ dividing $n(n-1)$, we write $\mathcal{V}_{n, \mathfrak{p}}=X_{1, \mathfrak{p}}+X_{2, \mathfrak{p}}$, and denote by $W_{1, \mathfrak{p}}, W_{2, \mathfrak{p}}$ the intersection points of these two components; these points may be singular points on the surface $\mathcal{V}_{n}$; we call $s_{1, \mathfrak{p}}, s_{2, \mathfrak{p}}$ respectively the number of blow-ups necessary to desingularize these points. The fibre of $\mathcal{V}_{n}^{\prime}$ consists of the two elliptic components $X_{1, \mathfrak{p}}, X_{2, \mathfrak{p}}$, linked by rational components $A_{1, \mathfrak{p}}, \ldots, A_{s_{1, \mathfrak{p}}, \mathfrak{p}}$ (lying above $\left.W_{1, \mathfrak{p}}\right), B_{1, \mathfrak{p}}, \ldots, B_{s_{2, \mathfrak{p}}, \mathfrak{p}}$ (lying above $W_{2, \mathfrak{p}}$ ).

At a prime $\mathfrak{p}$ in $\mathcal{O}_{n}^{\prime}$ dividing 2 , we write $\mathcal{V}_{n, \mathfrak{p}}=X_{0, \mathfrak{p}}+X_{1, \mathfrak{p}}+X_{2, \mathfrak{p}}+X_{3, \mathfrak{p}}$, where $X_{0, \mathfrak{p}}$ is a rational component, and $X_{2, \mathfrak{p}}$ is the elliptic component that dominates the special fibre of $E$ through the map $\psi_{2}$. Again, the intersection points $Y_{1, \mathfrak{p}}, Y_{2, \mathfrak{p}}, Y_{3, \mathfrak{p}}$ of the three elliptic components with the rational component may be singular points on $\mathcal{V}_{n}$; we will denote by $r_{\mathfrak{p}}, s_{\mathfrak{p}}, t_{\mathfrak{p}}$ the number of blow-ups needed to desingularize these points. Since the automorphism $\gamma$ permutes $X_{1, \mathfrak{p}}$ and $X_{2, \mathfrak{p}}$, we know that $r_{\mathfrak{p}}=s_{\mathfrak{p}}$. The fibre of $\mathcal{V}_{n}^{\prime}$ consists of the three elliptic components, linked to $X_{0, \mathfrak{p}}$ by rational components $A_{1, \mathfrak{p}}, \ldots, A_{r_{\mathfrak{p}}, \mathfrak{p}}$ (lying above $Y_{1, \mathfrak{p}}$ ), $B_{1, \mathfrak{p}}, \ldots, B_{r_{\mathfrak{p}, \mathfrak{p}}}$ (lying above $\left.Y_{2, \mathfrak{p}}\right)$, and $C_{1, \mathfrak{p}}, \ldots, C_{s_{\mathfrak{p}, \mathfrak{p}}}$ (lying above $Y_{3, \mathfrak{p}}$ ). All these rational components 
are numbered starting from the corresponding elliptic component and ending in $X_{2, \mathfrak{p}}$.

We shall determine a canonical divisor on $\mathcal{V}_{n}^{\prime}$, that is, a divisor $D=D_{1}^{0}+$ $\sum_{\mathfrak{p}} D_{\mathfrak{p}}$ such that $\omega_{\mathcal{V}_{n}^{\prime} / T_{n}} \simeq \mathcal{O}_{\mathcal{V}_{n}^{\prime}}(D)$. We write $D_{\mathfrak{p}}=\sum_{i} a_{i, \mathfrak{p}} X_{i, \mathfrak{p}}+E_{\mathfrak{p}}$, with the $a_{i, \mathfrak{p}}$ integer coefficients, and $E_{\mathfrak{p}}$ a sum of rational components coming from blow-ups. The advantage of working on a regular model is that we may use the adjunction formula for every component $X$ of a fibre $\mathcal{V}_{n, \mathfrak{p}}^{\prime}$ :

$$
2 g_{X}-2=\omega_{\mathcal{V}_{n}^{\prime} / T_{n}} \mid X+X^{2}=\left(D_{1}^{0}, X\right)+\left(D_{\mathfrak{p}}, X\right)+X^{2},
$$

to obtain a linear system of equations on the coefficients of $D_{\mathfrak{p}}$. By Proposition 9.2 , we only have to deal with the primes of bad reduction.

Let $\mathfrak{p}$ be an odd prime in $\mathcal{O}_{n}^{\prime}$ of bad reduction. We write $D_{\mathfrak{p}}=a_{1, \mathfrak{p}} X_{1, \mathfrak{p}}+$ $a_{2, \mathfrak{p}} X_{2, \mathfrak{p}}+\sum_{i} \alpha_{i} A_{i, \mathfrak{p}}+\sum_{j} \beta_{j} B_{j, \mathfrak{p}}$. The adjunction formula applied to every component of $\mathcal{V}_{n, \mathfrak{p}}^{\prime}$ yields a (degenerate) linear system of equations in the coefficients of $D$; solving this system we see that $D_{\mathfrak{p}}$ must be an integral multiple of the whole fibre $\mathcal{V}_{n, \mathfrak{p}}^{\prime}$.

In a prime $\mathfrak{p}$ dividing 2 , we write $D_{\mathfrak{p}}=\sum_{k=0}^{3} a_{k, \mathfrak{p}} X_{k, \mathfrak{p}}+\sum_{i} \alpha_{i} A_{i, \mathfrak{p}}+$ $\sum_{j} \beta_{j} B_{j, \mathfrak{p}}+\sum_{k} \gamma_{k} C_{k, \mathfrak{p}}$. The solutions of the linear system provided by the adjunction formula are

$$
\begin{array}{lll}
\alpha_{i}=a_{1, \mathfrak{p}}+i, & \beta_{j}=a_{2, \mathfrak{p}}-3 j, & \gamma_{k}=a_{3, \mathfrak{p}}+j, \\
a_{1, \mathfrak{p}}=a_{0}-r_{\mathfrak{p}}-1, & a_{2, \mathfrak{p}}=a_{0}+3\left(r_{\mathfrak{p}}+1\right), & a_{3, \mathfrak{p}}=a_{0}-s_{\mathfrak{p}}-1 .
\end{array}
$$

We may take $a_{0, \mathfrak{p}}=r_{\mathfrak{p}}+s_{\mathfrak{p}}+1$, and hence, up to integral multiples of the fibre:

$$
D_{\mathfrak{p}}=\left(r_{\mathfrak{p}}+s_{\mathfrak{p}}+1\right) X_{0, \mathfrak{p}}+s_{\mathfrak{p}} X_{1}+\left(s_{\mathfrak{p}}+4\left(r_{\mathfrak{p}}+1\right)\right) X_{2, \mathfrak{p}}+r_{\mathfrak{p}} X_{3}+E_{\mathfrak{p}},
$$

where $E_{\mathfrak{p}}=\sum_{i=1}^{r_{\mathfrak{p}}}\left(s_{\mathfrak{p}}+i\right) A_{i, \mathfrak{p}}+\sum_{i=1}^{s_{\mathfrak{p}}}\left(r_{\mathfrak{p}}+i\right) C_{i, \mathfrak{p}}+\sum_{j=1}^{r_{\mathfrak{p}}}\left(s_{\mathfrak{p}}+4\left(r_{\mathfrak{p}}+1\right)-\right.$ $3 j) B_{j, \mathfrak{p}}$. From now on, we shall denote by $F_{2}$ the sum of all the $D_{\mathfrak{p}}$ for $\mathfrak{p}$ dividing 2:

$$
F_{2}:=\sum_{\mathfrak{p} \mid 2}\left(\left(r_{\mathfrak{p}}+s_{\mathfrak{p}}+1\right) X_{0, \mathfrak{p}}+s_{\mathfrak{p}} X_{1}+\left(s_{\mathfrak{p}}+4\left(r_{\mathfrak{p}}+1\right)\right) X_{2, \mathfrak{p}}+r_{\mathfrak{p}} X_{3}+E_{\mathfrak{p}}\right) .
$$

We have thus seen that, for certain divisor $V$ on $\mathcal{V}_{n}$ coming from an ideal $I$ in $\mathcal{O}_{n}^{\prime}$ dividing $n(n-1)$,

$$
\omega_{\mathcal{V}_{n} / T_{n}} \simeq \mathcal{O}_{\mathcal{V}_{n}}\left(M_{1}+M_{2}+M_{3}+M_{4}+F_{2}+V\right) .
$$

In order to avoid the determination of the divisor $V$ coming from the base, we have imposed the condition defining the field $L_{n}$ at the beginning of the section. Then, the divisor $V$ becomes a principal ideal, and we can ignore it ${ }^{1}$.

\footnotetext{
${ }^{1}$ Anyway, the computations in Section 10 could be carried on without additional effort if we define $L_{n}=K\left(P_{1}, P_{3}\right)$ and take into account the (indeterminate) divisor $V$; we have chosen our definition of $L_{n}$ to simplify the expressions to be obtained later on.
} 
Theorem 9.4. The divisor $D_{1}=M_{1}+M_{2}+M_{3}+M_{4}+F_{2}$ is a canonical divisor on $\mathcal{V}_{n}^{\prime}$ :

$$
\omega_{\mathcal{V}_{n}^{\prime} / T_{n}} \simeq \mathcal{O}_{\mathcal{V}_{n}^{\prime}}\left(D_{1}\right)
$$

We note that for any automorphism $\sigma$ of $C_{n}, \sigma\left(D_{1}\right)$ is also a canonical divisor on $\mathcal{V}_{n}^{\prime}$. Let us take $D_{2}=\varphi_{01}\left(D_{1}\right)$. Putting $Q_{i}=\varphi_{01}\left(P_{i}\right), N_{i}=$ $\varphi_{01}\left(M_{i}\right)$, we have that $D_{2}=N_{1}+N_{2}+N_{3}+N_{4}+F_{2}$, since $\varphi_{01}$ leaves the components of the fibres at even primes invariant, as we have seen in Section 7. Since $Q_{i} \neq P_{j}$, the divisors $D_{1}$ and $D_{2}$ are disjoint on the generic fibre, so that we can use them to compute the self-intersection of the canonical sheaf.

Let us write $R_{1}=\psi_{2}\left(P_{1}\right)=\psi_{2}\left(P_{2}\right), R_{2}=\psi_{2}\left(P_{3}\right)=\psi_{2}\left(P_{4}\right), T_{1}=$ $\psi_{2}\left(Q_{1}\right)=\psi_{2}\left(Q_{2}\right), T_{2}=\psi_{2}\left(Q_{3}\right)=\psi_{2}\left(Q_{4}\right)$. We know that $\varphi_{01}$ induces the automorphism $\varphi_{01}^{E}=[-i]_{E}+(1,0)$ on $E$ through $\psi_{2}$. Thus $T_{k}=$ $[-i]_{E} R_{k}+(1,0)$, and $3\left(T_{j}-R_{k}\right)=(1,0)$ has exact order 2 ; in particular $T_{j} \neq R_{k}$. Let us denote by $R_{j, \mathfrak{p}}, T_{k, \mathfrak{p}}$ the reductions of the points $R_{j}, T_{k}$ modulo a prime $\mathfrak{p}$. It is clear that if these reductions do not coincide, the corresponding sections $M_{j}, N_{k}$ do not intersect on the fibre at $\mathfrak{p}$. If $\mathfrak{p}$ is an odd prime, the elliptic curve $E$ has good reduction at $\mathfrak{p}$ and everything reduces properly, so that $R_{j, \mathfrak{p}} \neq T_{k, \mathfrak{p}}$. If $\mathfrak{p} \mid 2$, the reduction of $\varphi_{01}^{E}$ is $[-i]_{E_{/ \ell \mathfrak{p}}}$, and we have $T_{1, \mathfrak{p}}=-T_{2, \mathfrak{p}}=[-i]_{E_{/ \ell \mathfrak{p}}} R_{1}=[i]_{E_{/ \ell \mathfrak{p}}} R_{2}$, so that again $R_{j, \mathfrak{p}} \neq T_{k, \mathfrak{p}}$. We have seen:

Proposition 9.5. The divisors $D_{1}^{0}=D_{1}-F_{2}$ and $D_{2}^{0}=D_{2}-F_{2}$ are disjoint.

In the following section we will need the following computation:

Lemma 9.6. $\left(F_{2}, F_{2}\right)=-\sum_{\mathfrak{p} \mid 2}\left(10 r_{\mathfrak{p}}+s_{\mathfrak{p}}+11\right) \log \sharp \ell_{\mathfrak{p}}$.

Proof. We have $F_{2}=\sum_{\mathfrak{p}} D_{\mathfrak{p}}$, and $\left(D_{\mathfrak{p}}, D_{\mathfrak{p}}\right)=-\left(10 r_{\mathfrak{p}}+s_{\mathfrak{p}}+11\right) \log \sharp \ell_{\mathfrak{p}}$ follows from the equalities (where the factor $\log \sharp \ell_{\mathfrak{p}}$ is skipped):

$$
\begin{aligned}
& D_{\mathfrak{p}}=\left(r_{\mathfrak{p}}+s_{\mathfrak{p}}+1\right) X_{0, \mathfrak{p}}+s_{\mathfrak{p}} X_{1}+\left(s_{\mathfrak{p}}+4\left(r_{\mathfrak{p}}+1\right)\right) X_{2, \mathfrak{p}}+r_{\mathfrak{p}} X_{3}+E_{\mathfrak{p}}, \\
& E_{\mathfrak{p}}=\sum_{i=1}^{r_{\mathfrak{p}}}\left(s_{\mathfrak{p}}+i\right) A_{i, \mathfrak{p}}+\sum_{i=1}^{s_{\mathfrak{p}}}\left(r_{\mathfrak{p}}+i\right) C_{i, \mathfrak{p}} \\
& \quad+\sum_{j=1}^{r_{\mathfrak{p}}}\left(s_{\mathfrak{p}}+4\left(r_{\mathfrak{p}}+1\right)-3 j\right) B_{j, \mathfrak{p}}, \\
& \left(\sum_{i=1}^{r_{\mathfrak{p}}}(s+i) A_{i, \mathfrak{p}}\right)^{2}=-\left(r_{\mathfrak{p}}+s_{\mathfrak{p}}+1\right)^{2}-s_{\mathfrak{p}}^{2}+r_{\mathfrak{p}}+1,
\end{aligned}
$$$$
\left(\sum_{i=1}^{s_{\mathfrak{p}}}(r+i) C_{i, \mathfrak{p}}\right)^{2}=-\left(r_{\mathfrak{p}}+s_{\mathfrak{p}}+1\right)^{2}-r_{\mathfrak{p}}^{2}+s_{\mathfrak{p}}+1,
$$ 


$$
\begin{aligned}
& \left(\sum_{j=1}^{r_{\mathfrak{p}}}\left(s_{\mathfrak{p}}+4\left(r_{\mathfrak{p}}+1\right)-3 j\right) B_{j, \mathfrak{p}}\right)^{2}=-17 r_{\mathfrak{p}}^{2}-10 r_{\mathfrak{p}} s_{\mathfrak{p}}-25 r_{\mathfrak{p}}-2 s_{\mathfrak{p}}^{2}-10 s_{\mathfrak{p}}-8 \\
& \left(E_{\mathfrak{p}}, E_{\mathfrak{p}}\right)=\left(\sum_{i=1}^{r_{\mathfrak{p}}}(s+i) A_{i, \mathfrak{p}}\right)^{2}+\left(\sum_{j=1}^{r_{\mathfrak{p}}}\left(s_{\mathfrak{p}}+4\left(r_{\mathfrak{p}}+1\right)-3 j\right) B_{j, \mathfrak{p}}\right)^{2} \\
& +\left(\sum_{i=1}^{s_{\mathfrak{p}}}(r+i) C_{i, \mathfrak{p}}\right)^{2} \\
& =-20 r_{\mathfrak{p}}^{2}-14 r_{\mathfrak{p}} s_{\mathfrak{p}}-28 r_{\mathfrak{p}}-5 s_{\mathfrak{p}}^{2}-13 s_{\mathfrak{p}}-8, \\
& \left(X_{0, \mathfrak{p}}, E_{\mathfrak{p}}\right)=3\left(r_{\mathfrak{p}}+s_{\mathfrak{p}}\right)+4, \quad\left(X_{0, \mathfrak{p}}, X_{0, \mathfrak{p}}\right)=-3, \\
& \left(X_{1, \mathfrak{p}},\left(r_{\mathfrak{p}}+s_{\mathfrak{p}}+1\right) X_{0, \mathfrak{p}}+E_{\mathfrak{p}}\right)=s_{\mathfrak{p}}+1, \quad\left(X_{1, \mathfrak{p}}, X_{1, \mathfrak{p}}\right)=-1, \\
& \left(X_{2, \mathfrak{p}},\left(r_{\mathfrak{p}}+s_{\mathfrak{p}}+1\right) X_{0, \mathfrak{p}}+E_{\mathfrak{p}}\right)=s_{\mathfrak{p}}+4 r_{\mathfrak{p}}+1, \quad\left(X_{2, \mathfrak{p}}, X_{2, \mathfrak{p}}\right)=-1, \\
& \left(X_{3, \mathfrak{p}},\left(r_{\mathfrak{p}}+s_{\mathfrak{p}}+1\right) X_{0, \mathfrak{p}}+E_{\mathfrak{p}}\right)=r_{\mathfrak{p}}+1, \quad\left(X_{3, \mathfrak{p}}, X_{3, \mathfrak{p}}\right)=-1 \text {. }
\end{aligned}
$$

\section{A formula for Arakelov self-intersection.}

We will now give an explicit expression for the Arakelov self-intersection of the canonical sheaf of the arithmetic surfaces $\mathcal{V}_{n}$. Since this self-intersection is unaltered after a blow-up, we can compute it with the canonical divisors on $\mathcal{V}_{n}^{\prime}$ which we have found in previous section.

First of all, we must extend the isomorphism (2) to an Arakelov isomorphism. The curvature of $\omega_{\mathcal{V}_{n}^{\prime} / T_{n}}$ and $\mathcal{O}_{\mathcal{V}_{n}^{\prime}}\left(D_{j}\right)$ being the same, it will be enough to add some vertical Arakelov components to the divisors $D_{1}, D_{2}$. Let us write:

$$
\bar{\omega}_{\mathcal{V}_{n}^{\prime} / T_{n}} \simeq \overline{\mathcal{O}}_{\mathcal{V}_{n}^{\prime}}\left(D_{j}+\sum_{\sigma: L_{n} \rightarrow \mathbb{C}} r_{j, \sigma} C_{n, \sigma}\right) .
$$

We have taken $D_{2}=\varphi_{01}\left(D_{1}\right)$, and $\varphi_{01}$ is defined over $K_{n}$, so that $r_{1, \sigma}=r_{2, \sigma}$ for every immersion $\sigma: L_{n} \rightarrow \mathbb{C}$, and the divisors $D_{1}, D_{2}$ are Arakelov equivalent. Moreover, this equality yields:

$$
\left(\bar{\omega}_{\mathcal{V}_{n}^{\prime} / T_{n}}, \bar{\omega}_{\mathcal{V}_{n}^{\prime} / T_{n}}\right)_{\mathrm{Ar}}=\left(D_{1}, D_{2}\right)_{\mathrm{Ar}}+8 \sum_{\sigma} r_{1, \sigma}
$$

We can determine this sum using the arithmetic adjunction formula (cf. [Sz85]), which in our case gives: $\left(\bar{\omega}_{\mathcal{V}_{n}^{\prime} / T_{n}}, \overline{\mathcal{O}}_{\mathcal{V}_{n}^{\prime}}\left(M_{j}\right)\right)_{\mathrm{Ar}}=-\left(M_{j}, M_{j}\right)_{\mathrm{Ar}}$. Combining this relation with the first equality we obtain

$$
\sum_{\sigma} r_{1, \sigma}=-\left(M_{j}, M_{j}\right)_{\mathrm{Ar}}-\left(M_{j}, D_{1}\right)_{\mathrm{Ar}},
$$


and summing up for all $M_{j}$ 's:

$$
4 \sum_{\sigma} r_{1, \sigma}=-\sum_{j=1}^{4}\left(M_{j}, M_{j}\right)_{\mathrm{Ar}}-\left(D_{1}, D_{1}\right)_{\mathrm{Ar}}+\left(F_{2}, D_{1}\right)_{\mathrm{Ar}} .
$$

The self-intersection of the $M_{j}$ 's can be eliminated using the equality ( $D_{1}^{0}$, $\left.D_{1}^{0}\right)_{\mathrm{Ar}}=\sum_{j=1}^{4}\left(M_{j}, M_{j}\right)_{\mathrm{Ar}}+2 \sum_{j<k}\left(M_{j}, M_{k}\right)_{\mathrm{Ar}}$ :

$$
4 \sum_{\sigma} r_{1, \sigma}=-2\left(D_{1}, D_{1}\right)_{\mathrm{Ar}}+3\left(D_{1}, F_{2}\right)_{\mathrm{Ar}}-\left(F_{2}, F_{2}\right)_{\mathrm{Ar}}+2 \sum_{j<k}\left(M_{j}, M_{k}\right)_{\mathrm{Ar}} .
$$

If we now take into account that $\left(D_{1}, D_{1}\right)_{\mathrm{Ar}}=\left(D_{1}, D_{2}\right)_{\mathrm{Ar}}$ since the divisors $D_{1}, D_{2}$ are Arakelov equivalent, we obtain that:

$$
\begin{aligned}
\left(\bar{\omega}_{\mathcal{V}_{n}^{\prime} / T_{n}}, \bar{\omega}_{\mathcal{V}_{n}^{\prime} / T_{n}}\right)_{\mathrm{Ar}}= & -3\left(D_{1}, D_{2}\right)_{\mathrm{Ar}}+4 \sum_{j<k}\left(M_{j}, M_{k}\right)_{\mathrm{Ar}} \\
& +6\left(D_{1}, F_{2}\right)_{\mathrm{Ar}}-2\left(F_{2}, F_{2}\right)_{\mathrm{Ar}} .
\end{aligned}
$$

We will now give more concrete expressions for the terms in the right of the previous equality. We must introduce some notations. For every $\sigma: L_{n} \rightarrow \mathbb{C}$, we denote by $P_{j}^{\sigma}$ (resp. $Q_{k}^{\sigma}$ ) the points given by the sections $M_{j}\left(\right.$ resp. $N_{k}$ ) on the fibre $C_{n, \sigma}$ of $\mathcal{V}_{n}$ at $\sigma$.

Lemma 10.1. Let $G$ be the Green function of the Riemann surface given by the curve $C_{n}$. We have:

$$
\left(D_{1}, D_{2}\right)_{\mathrm{Ar}}=\sum_{\sigma} \sum_{j, k=1}^{4} \log G\left(P_{j}^{\sigma}, Q_{k}^{\sigma}\right)+2\left(D_{1}^{0}, F_{2}\right)_{\mathrm{Ar}}+\left(F_{2}, F_{2}\right)_{\mathrm{Ar}} .
$$

Proof. We have:

$$
\left(D_{1}, D_{2}\right)_{\mathrm{Ar}}=\left(D_{1}^{0}, D_{2}^{0}\right)_{\mathrm{Ar}}+\left(D_{1}^{0}, F_{2}\right)_{\mathrm{Ar}}+\left(D_{2}^{0}, F_{2}\right)_{\mathrm{Ar}}+\left(F_{2}, F_{2}\right)_{\mathrm{Ar}} .
$$

By Proposition 9.5, the first term equals $\sum_{\sigma} \sum_{j, k=1}^{4} \log G\left(P_{j}^{\sigma}, Q_{k}^{\sigma}\right)$. The two intermediate terms are equal since $D_{2}^{0}=\varphi_{01}\left(D_{1}^{0}\right)$, and $F_{2}$ is invariant through $\varphi_{01}$.

\section{Lemma 10.2.}

$$
\begin{aligned}
\sum_{j<k}\left(M_{j}, M_{k}\right)_{\mathrm{Ar}}= & \sum_{\sigma} \sum_{j<k} \log G\left(P_{j}^{\sigma}, P_{k}^{\sigma}\right) \\
& +2 \sum_{\mathfrak{p} \mid 2}\left(M_{1}, M_{2}\right)_{\mathfrak{p}}+2 \sum_{\mathfrak{p} \mid 3}\left(M_{1}, M_{3}+M_{4}\right)_{\mathfrak{p}} .
\end{aligned}
$$

Proof. We calculate the finite part of this intersection. If $\mathfrak{p} \mid 2$, then

$$
\left(M_{1}, M_{3}\right)_{\mathfrak{p}}=\left(M_{1}, M_{4}\right)_{\mathfrak{p}}=\left(M_{2}, M_{3}\right)_{\mathfrak{p}}=\left(M_{2}, M_{4}\right)_{\mathfrak{p}}=0,
$$

because the images of these pairs of points through the map $\psi_{2}$ are different. On the other hand, the pair of points $M_{1}, M_{2}$ are conjugated to the pair $M_{3}, M_{4}$ over $\mathbb{Q}$ by Lemma 9.1 , and thus $\sum_{\mathfrak{p} \mid 2}\left(M_{1}, M_{2}\right)_{\mathfrak{p}}=\sum_{\mathfrak{p} \mid 2}\left(M_{3}, M_{4}\right)_{\mathfrak{p}}$. 
We now look at an odd prime $\mathfrak{p}$ dividing $n(n-1)$. We know from Lemma 9.3 that two of the sections $M_{1}, M_{2}, M_{3}, M_{4}$ intersect the component $X_{1, \mathfrak{p}}$ of the fibre $\mathcal{V}_{n, \mathfrak{p}}$, and the other two intersect the component $X_{2, \mathfrak{p}}$. Moreover, $M_{1}$ and $M_{2}$ must intersect different components, since $M_{2}=$ $\varphi_{12}\left(M_{1}\right), X_{2, \mathfrak{p}}=\varphi_{12}\left(X_{1, \mathfrak{p}}\right)$. The same assertion is true for $M_{3}, M_{4}$. The $M_{j}$ are contained in the smooth part of $\mathcal{V}_{n}$, so that we must have:

$$
\left(M_{1}, M_{2}\right)_{\mathfrak{p}}=\left(M_{3}, M_{4}\right)_{\mathfrak{p}}=0 .
$$

Now, $\left(M_{1}, M_{3}+M_{4}\right)_{\mathfrak{p}}=\left(M_{2}, M_{3}+M_{4}\right)_{\mathfrak{p}}$, again because $M_{2}=\varphi_{12}\left(M_{1}\right)$, $M_{4}=\varphi_{12}\left(M_{3}\right)$. Note that $\psi_{2}\left(P_{1}\right)=R_{1}$ is a 3 -torsion point distinct from $\psi_{2}\left(P_{3}\right)=\psi_{2}\left(P_{4}\right)=R_{2}=2 R_{1}$. Hence, if these sections do intersect at $\mathfrak{p}$, the points $R_{1, \mathfrak{p}}, R_{2, \mathfrak{p}}$ must specialize both to 0 . This can only happen at those primes with residue characteristic 3 .

Putting all the above equalities together, and taking into account Lemma 9.6 we obtain:

Theorem 10.3. The self-intersection of the Arakelov dualizing sheaf of the arithmetic surface $\mathcal{V}_{n}^{\prime}$ can be expressed as:

$$
\begin{aligned}
\left(\bar{\omega}_{\mathcal{V}_{n}^{\prime} / T_{n}}, \bar{\omega}_{\mathcal{V}_{n}^{\prime} / T_{n}}\right)_{\mathrm{Ar}}= & 4 \sum_{\sigma} \sum_{j<k} \log G\left(P_{j}^{\sigma}, P_{k}^{\sigma}\right) \\
& -3 \sum_{\sigma} \sum_{j, k=1}^{4} \log G\left(P_{j}^{\sigma}, Q_{k}^{\sigma}\right) \\
& +8 \sum_{\mathfrak{p} \mid 2}\left(M_{1}, M_{2}\right)_{\mathfrak{p}}+8 \sum_{\mathfrak{p} \mid 3}\left(M_{1}, M_{3}+M_{4}\right)_{\mathfrak{p}} \\
& -\sum_{\mathfrak{p} \mid 2}\left(10 r_{\mathfrak{p}}+s_{\mathfrak{p}}+11\right) \log \sharp \ell_{\mathfrak{p}} .
\end{aligned}
$$

Acknowledgements. I wish to thank Prof. P. Bayer for her helpful comments during the preparation of this work, and also the referee for his/her useful suggests.

\section{References}

[BBBC099] C. Batut, K. Belabas, D. Bernardi, H. Cohen and M. Olivier, PARI, http://www.parigp-home.de/.

[BLR90] S. Bosch, W. Lütkebohmert and M. Raynaud, Néron Models, Ergebnisse der Mathematik, 21, Springer, Berlin, 1990, MR 91i:14034, Zbl 0705.14001.

[Bo-M-M90] J.B. Bost, J.-F. Mestre and L. Moret-Bailly, Sur le calcul explicite des "classes de Chern" des surfaces arithmétiques de genre 2, Astérisque, 183 (1990), 69-105, MR 92g:14018a, Zbl 0731.14017.

[Ca90] P. Cartier et al., eds, The Grothendieck Festschrift, Vol. III, Progress in Mathematics, 88, Birkhäuser, Boston, 1990, MR 92b:00052, Zbl 0717.00010. 
[De85] P. Deligne, Preuve des conjectures de Tate et Shafarevitch (d'après G. Faltings), Séminaire Bourbaki, exp. 616, Astérisque, 121-122 (1985), 25-41, MR 87c:11026, Zbl 0591.14026.

[De-Mu69] P. Deligne and D. Mumford, The irreducibility of the space of curves of given genus, Publ. Math. I.H.E.S., 36 (1969), 75-109, MR 41 \#6850, Zbl 0181.48803.

[Ds81] M. Deschamps, Réduction semi-stable, Séminaire sur les pinceaux de courbes de genre au moins deux, Astérisque, 86 (1981), 1-34, Zbl 0505.14008.

[Fa84] G. Faltings, Calculus on arithmetic surfaces, Ann. of Math., 119 (1984), 387-424, MR 86e:14009, Zbl 0559.14005.

[Gu01] J. Guàrdia, Explicit geometry on a family of genus 3 curves, J. London Math. Soc., 64(2) (2001), 299-310, MR 2002f:11075.

[La88] S. Lang, Introduction to Arakelov Theory, Springer, New York, 1988, MR 89m:11059, Zbl 0667.14001.

[Li69] J. Lipman, Rational singularities, Publ. Math. I.H.E.S., 36 (1969), 195-279, MR 43 \#1986, Zbl 0181.48903.

[MB85] L. Moret-Bailly, Métriques permises, Séminaire sur les pinceaux arithmétiques, Astérisque, 127 (1985), 29-87, CMP 1801935.

[Mo96] A. Moriwaki, Lower bound of self-intersection of dualizing sheaves on arithmertic surfaces with reducible fibres, Math. Ann., 305 (1996), 183-190, MR 97b:14025, Zbl 0845.14015.

[Ra85] M., Raynaud, Hauteurs et isogénies, Séminaire sur les pinceaux arithmétiques: La conjecture de Mordell, Astérisque, 127 (1985), 199-234, CMP 1801923.

[Ra90] ㄴ. MR 92m:14025, Zbl 0722.14013.

[Se-Ta68] J.P. Serre and J. Tate, Good reduction of abelian varieties, Ann. of Math., 88 (1968), 492-517, MR 38 \#4488, Zbl 0217.05201.

[Si85] J. Silverman, The Arithmetic of Elliptic Curves, Graduate Texts in Math., 106, Springer-Verlag, New York, 1986, MR 87g:11070, Zbl 0585.14026.

[Sz85] L. Szpiro, Degrés, intersections, hauteurs, Séminaire sur les pinceaux arithmétiques: La conjecture de Mordell, Astérisque, 127 (1985), 11-28, CMP 1801917.

Received November 22, 2000 and revised January 14, 2003. The author was partially supported by MCYT BFM2000-0627.

Dept. Matemàtica Aplicada IV

Escola Universitària Politècnica de Vilanova i la Geltrú

Universitat Politècnica de CATAlunya

E-08800 Vilanova i la Geltrú

Barcelona - Catalunya

SPAIN

E-mail address: guardia@mat.upc.es 